\title{
Medicina traslacional
}

\author{
Miguel Cruz-López
}

Coeditor de Gaceta Médica de México

\section{Introducción}

La definición de medicina traslacional (MT) no es clara, pero representa un enfoque novedoso cuyo fin es procurar al paciente un beneficio en el lapso de tiempo más corto posible. La investigación biomédica se orienta a descubrir nuevas herramientas diagnósticas y nuevos tratamientos a través del enfoque altamente especializado multidisciplinario. La MT utiliza los descubrimientos científicos para generar herramientas nuevas que mejoren la salud humana al reducir la incidencia de la morbilidad y la mortalidad. Fomenta la investigación multidireccional, la integración de la investigación básica, la investigación orientada al diagnóstico de la enfermedad y su tratamiento.

El término MT se acuñó en la década de 1990, pero el uso del concepto no empezó hasta principios del año 2000. En 2003, la investigación clínica del Instituto de Medicina (EE. UU.) describió la terminología y el modelo de investigación traslacional como un proceso a partir de la ciencia básica dirigido a la investigación clínica para establecer una política de salud pública, entre otras perspectivas. La Universidad de Cincinnati (Children's Hospital Medical Center), define a la MT como la encargada de transformar los descubrimientos científicos en nuevos instrumentos y aplicaciones clínicas que mejoran la salud de las personas (Transforming Translation - Harnessing Discovery for Patient and Public Benefit, 2007). El instituto The Wellcome Trust, en Gran Bretaña, amplía la definición de MT y la convierte en estudios de innovación, con nuevos productos para la industria médica, farmacéutica o biotecnológica; es decir, traslada los descubrimientos a productos comerciales lo antes posible. El Instituto Scripps, en Florida, incorpora otro elemento distintivo a este mismo proceso al reunir investigadores procedentes del mundo académico e industrial, médicos y especialistas diversos para conformar plataformas tecnológicas en las que los bioinformáticos y los expertos en genómica, farmacocinética y clínica sumen sus conocimientos para estudios de diversas patologías. En los Institutos $\mathrm{Na}$ cionales de Salud ( $\mathrm{NIH}$, por sus siglas en inglés $\mathrm{NIH}$ ), el concepto de MT adquiere un sentido más avanzado. Se trata de ir desde el laboratorio a la cama del paciente, lo que marca la tendencia en la investigación biomédica del primer cuarto del siglo XXI. De lo que se trata es de catalizar la unión de los nuevos conocimientos generados en los laboratorios básicos con los desarrollos, observaciones, tecnologías e innovaciones producidos en todos los ámbitos de investigación multidisciplinaria. Para ello, los NIH promovieron un amplio consorcio en el que participan más de 60 instituciones, entre universidades, empresas y hospitales, con el objetivo de compartir conocimientos, tecnologías e incluso proyectos tanto para el desarrollo de nuevas terapias como para abordar mejoras o nuevas visiones, fruto de aproximaciones multidisciplinarias, con un aporte de 10,000 millones de dólares a los centros de MT. En esa misma línea, el Howard Hughes Medical Institute, una de las más prestigiosas instituciones de investigación en los EE.UU., ha puesto en marcha programas de financiamiento para investigadores clínicos que participen activamente en proyectos de investigación básica. La revista Science Translational Medicine promueve y divulga la MT, lo que permite a los investigadores de manera eficiente aplicar nuevos resultados en diferentes campos de conocimiento. Los científicos en el

\footnotetext{
Correspondencia:

Miguel Cruz-López

Avda. Cuauhtémoc, 330, Bloque B

Col. Doctores

C.P. 06725, Ciudad de México, México

E-mail: mcruzl@yahoo.com
}

Fecha de recepción: 29-09-2017

Fecha de aceptación: 02-10-2017

DOI:10.24875/GMM.M17000016
Gac Med Mex. 2017;153:547-549

Contents available at PubMed www.gacetamedicademexico.com 
laboratorio utilizan las muestras clínicas para el diagnóstico, mediante el análisis de biomarcadores o para esclarecer la diferencia entre el estado normal y el patológico. Investigadores de la Universidad de California en Irvine describieron, en la revista American Journal of Translational Research, que las observaciones de los investigadores clínicos se vinculen con las investigaciones básicas para encontrar los mecanismos alterados en el paciente.

\section{Logros de la medicina traslacional}

La Universidad Johns Hopkins y un grupo de investigadores demostraron que el losartán, medicamento ampliamente utilizado en el tratamiento de la hipertensión arterial, puede prevenir el desarrollo de los aneurismas aórticos encontrados en ratones diseñados para reproducir el síndrome de Marfan, una enfermedad genética que afecta el sistema conectivo. Este medicamento se ha probado ahora como terapia en un grupo de niños con este síndrome y se ha encontrado que inhibe el desarrollo de estas anomalías potencialmente letales en la aorta. En el campo tecnológico se han desarrollado algoritmos sofisticados de procesamiento de imágenes de alta resolución para detectar y localizar tumores de próstata en etapa temprana. Otra área de investigación fue la vacuna para la meningitis B. El grupo dirigido por Rino Rappuoli, de IRIS, Chiron SpA, en Siena (Italia), identificó una vacuna candidata usando un enfoque traslacional llamado «vacunología inversa», por medio de la secuencia del genoma. En el campo de la oftalmología, muchos pacientes con glaucoma se deben administrar gotas oculares numerosas veces durante el día; en la Universidad de Yale se han desarrollado microesferas que contienen el fármaco maleato de timolol, que se puede inyectar en un punto en el ojo, desde el que las microesferas secretan cantidades controladas de timolol durante más de un mes. Las nanopartículas de oro o nanoshells desarrolladas en la Universidad de Texas para detectar células cancerosas por espectroscopia de fluorescencia son otro ejemplo.

\section{Investigación biomédica}

Los científicos usan la MT para ampliar su base de conocimiento para descubrir maneras de prevenir enfermedades y desarrollar productos útiles, como medicamentos y procedimientos, para tratar y curar enfermedades. La investigación biomédica requiere el aporte y la participación de equipos humanos con habilidades diferentes, como médicos, veterinarios, informáticos, ingenieros, técnicos, investigadores y una variedad de científicos de los diferentes campos de las ciencias de la vida. Se trata de creencias o teorías que pueden ser probadas o refutadas a través de observaciones y experimentación, primero en animales cuyas características representen mejor el proceso de enfermedad de los seres humanos. Casi todos los avances médicos importantes en este siglo se han basado en la investigación en animales.

\section{Investigación de la traducción en farmacogenómica}

La capacidad de diseñar con mayor precisión fármacos de interés médico y probarlos en modelos animales para su posterior utilización en los pacientes es otro ejemplo de la aplicación de la MT. Diversos procesos patológicos, en combinación con la farmacogenómica, se utilizan para comprender cómo la variación genómica produce confusión al personal médico en muchas ocasiones, cuando no se tiene la respuesta esperada con el tratamiento aplicado al paciente.

\section{Investigación traslacional de enfermedades cardiovasculares}

Para la aplicación de la investigación traslacional en la salud y el cuidado de pacientes con enfermedades congénitas se han creado grupos de colaboración a través de consorcios que interactúan permanentemente. El Consorcio de Desarrollo Cardiovascular colabora con equipos de investigación utilizando modelos animales para profundizar en el conocimiento de las redes de regulación transcripcional que rigen el desarrollo del corazón. Los investigadores clínicos reclutan a los niños para el estudio de los genes causales y para evaluar los efectos de la variación genética en pacientes con enfermedades cardiacas congénitas. Los consorcios complementan la Red de Investigación de Terapia Celular Cardiovascular, para promover y acelerar la investigación clínica en la evaluación de nuevas estrategias de tratamiento de estas enfermedades.

\section{Medicina regenerativa traslacional}

Es el campo de la medicina con el potencial de curar los tejidos y los órganos dañados, ofreciendo 
soluciones y esperanza a los pacientes que hoy no están más allá de la reparación. La medicina regenerativa emplea la ingeniería de tejidos y la biología molecular para reemplazar o regenerar células, tejidos u órganos humanos. Utiliza fármacos dirigidos a moléculas específicas (p. ej., proteínas) en la superficie o dentro de células para detener el crecimiento y la diseminación de células cancerosas, limitando al mismo tiempo el daño a las células normales. Las terapias dirigidas emplean diferentes tipos de fármacos, y cada medicamento funciona de manera diferente. El enfoque es amplio y utiliza los avances en la tecnología de células madre para la atención clínica.

\section{Política basada en la evidencia}

La política basada en la evidencia de MT promueve un punto de vista más racional, riguroso y sistemático para la salud. La política basada en la evidencia se fundamenta en la premisa de que las decisiones de política deberían estar mejor sustentadas por las pruebas disponibles, e incluir además un análisis racional. El nuevo rumbo debe vincular las evidencias científicas a la toma de decisiones para hacer más rápida su utilización en los pacientes que no tienen otras alternativas terapéuticas.

\section{Conclusiones y retos}

La información disponible converge en la idea de que la MT puede ser de mucha utilidad para facilitar la llegada de nuevas herramientas diagnósticas y terapéuticas generadas desde la ciencia básica a la clínica, o de los hallazgos clínicos al conocimiento básico. Por ejemplo, el desarrollo de un nuevo fármaco suele tomar de 10 a 14 años para su uso en los pacientes y requiere una inversión que asciende a decenas de millones de pesos. Con la innovación de nuevas técnicas genómicas y proteómicas está cambiando el pensamiento para entender mejor la llamada MT. En la actualidad se prevé un futuro en la investigación biomédica que la hará más precisa en patologías de diferentes áreas, como la oncología y las enfermedades infecciosas, cardiovasculares y metabólicas, entre otras. Es urgente que el laboratorio, los estudios de gabinete y los equipos multidisciplinarios interactúen y analicen de manera integral al paciente lo antes posible, con la finalidad de hacer un diagnóstico temprano, instaurar un tratamiento apropiado (medicina de precisión) y, en especial, avanzar en la prevención para hacer de la medicina una nueva forma de abordar las patologías que afectan a la humanidad.

Los editores de la Gaceta Médica de México, Dr. Alejandro Treviño Becerra (editor), Dr. Francisco Espinosa Larrañaga (coeditor) y el que suscribe, invitan a los académicos y profesionales de la salud para promover en nuestro país el ejercicio de la MT y hacer de la investigación una aplicación para el paciente; al mismo tiempo, ponemos a su disposición las páginas de esta revista para divulgar sus experiencias y resultados de investigación en MT.

\section{Literatura recomendada}

Florez JC. The pharmacogenetics of metformin. Diabetologia. 2017. doi: 10.1007/s00125-017-4335-y. [Epub ahead of print]

Frau $\mathrm{F}$, Crowther $\mathrm{D}$, Ruetten $\mathrm{H}$, et al. Type-2 diabetes-associated variants with cross-trait relevance: post-GWAs strategies for biological function interpretation. Mol Genet Metab. 2017;121:43-50.

Littman $\mathrm{BH}$, Di Mario L, Plebani M, et al. What's next in translational medicine? Clin Sci (Lond). 2007;112:217-27.

Visscher PM, Wray NR, Zhang Q, et al. 10 Years of GWAS discovery: biology, function, and translation. Am J Hum Genet. 2017;101:5-22.

Wehling M. Translational medicine: science or wishful thinking? J Transl Med. 2008;6:31.

Wu Y, Jing R, Dong Y, et al. Functional annotation of sixty-five type-2 diabetes risk SNPs and its application in risk prediction. Sci Rep. 2017;6:43709. 